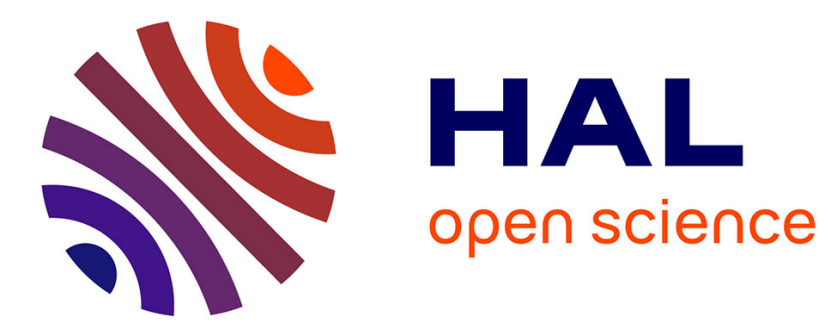

\title{
On the propagation of wireless signals on board ships
}

Andrea Mariscotti, Massimiliano Sassi, Andrea Qualizza, Matteo Lenardon

\section{To cite this version:}

Andrea Mariscotti, Massimiliano Sassi, Andrea Qualizza, Matteo Lenardon. On the propagation of wireless signals on board ships. 2010 IEEE Instrumentation \& Measurement Technology Conference Proceedings, May 2010, Austin, United States. pp.1418-1423, 10.1109/IMTC.2010.5488252 . hal03266580

\section{HAL Id: hal-03266580 \\ https://hal.science/hal-03266580}

Submitted on 21 Jun 2021

HAL is a multi-disciplinary open access archive for the deposit and dissemination of scientific research documents, whether they are published or not. The documents may come from teaching and research institutions in France or abroad, or from public or private research centers.
L'archive ouverte pluridisciplinaire HAL, est destinée au dépôt et à la diffusion de documents scientifiques de niveau recherche, publiés ou non, émanant des établissements d'enseignement et de recherche français ou étrangers, des laboratoires publics ou privés. 


\section{On the propagation of wireless signals on board ships}

\author{
Andrea Mariscotti \\ Dept. of Electrical Engineering \\ University of Genova \\ Genova, Italy
}

\author{
Massimiliano Sassi, Andrea Qualizza, Matteo Lenardon \\ Fincantieri S.p.A. \\ Genova, Italy
}

\begin{abstract}
The characterization of the wireless channel on board a cruise ship is considered by means of frequency domain measurements of received power and propagation attenuation. This electromagnetic environment is rather new and is characterized by abundance of reflecting surfaces and diffracting elements, so that a significant difference with respect to more common residential and office environments has been found. The measurement setup is simple and easy to position and operate. The results consist of corrected path loss laws for halls and corridors and the quantification of scattering influence.
\end{abstract}

Keywords-Indoor propagation channel, Path loss model, Wireless propagation

\section{INTRODUCTION}

There are several services and functions that may require a wireless connection on board ships; besides the interconnection of distributed devices for environmental monitoring, fire alarm and anti-intrusion systems, for passenger ships other services may extend to passenger information systems and support to personal mobile terminals, such as phones, netbooks, laptops, etc. The on board electromagnetic environment is rather new, while the most known and studied environments are offices, malls, schools and campus areas, characterized by (partially) absorbing materials, quite large spaces, medium height to high ceilings. For these environments several models have been proposed (deterministic and statistic) and there are databases of parameters and experimental results [1].

Deterministic models apply an electromagnetic simulation tool, such as ray tracing techniques, to obtain nearly exact propagation characteristics for a specified geometry, based on the physics of the propagation mechanisms such as path loss, reflection, diffraction, and scattering. The base path loss of free space is such that the power attenuation is proportional to the square of distance. Ray tracing models make an exact computation; the accuracy of ray tracing models relies on the accuracy and details of the site representation and materials properties [2]. The major advantage of these models is that they offer great accuracy with site-specific results. However, these models have some disadvantages:

- the topographical, geometrical and materials data are always tied to a particular site and thus a huge amount of such data is required in order to obtain a comprehensive set of different propagation scenarios;

- they are usually computationally intensive, especially when the environment is complex.
Numerous channel models for the $\mathrm{GHz}$ range, and in particular for Ultra Wide Band (UWB) signals, have been developed as deterministic models. For example, in [3]-[7] the ray tracing approach is used to model the received signal as a superposition of rays.

Statistical models are normally less complex numerically than deterministic models and can provide sufficiently accurate channel information. Statistical models attempt to generate synthetic channel responses that are representative of real propagation channels. Typically, such models can be tuned to various propagation environments by setting appropriate values for model parameters. Note that fixed parameter settings don't produce identical results for each simulation run, since stochastic variability is used within a fixed environment type.

Statistical models may be constructed on the basic principles of wave propagation and by assuming a statistical distribution of the channel parameters, and computing the required statistical moments from the data collected from the real-time measurements. This category of models has the ability to provide accurate statistical information, without the complexity of detailed deterministic approaches. Several statistical-based UWB channels have been proposed recently. Ghassemzadeh et al. [8] show a model based on the results of frequency domain channel measurements in the 4.375-5.625 $\mathrm{GHz}$ range in several residential environments; a path loss model and a second-order autoregressive model are proposed for the frequency response of the UWB indoor channel. Foerster and $\mathrm{Li}$ [9] propose a statistical-based multipath channel model based upon measurements collected in a condominium in the $2-8 \mathrm{GHz}$ range. Three channel models have been considered: the Rayleigh tap delay line model, the $\mathrm{D}-\mathrm{K}$ model and the Saleh-Valenzuela (S-V) model. The comparisons showed that the $\mathrm{S}-\mathrm{V}$ model gives the best fit to the measured channel characteristics such as the mean excess delay, mean RMS delay spread and the mean number of significant paths within $10 \mathrm{~dB}$ of the peak multipath arrival. In addition, the log-normal distribution was proposed to model the amplitude distribution, which was shown to give a better fit than the Rayleigh distribution. Chong et al. [10]-[13] incorporate the observed clustering of multipath components (MPCs) in their model; measurement results were obtained in the 3-10 GHz range in various types of apartments in Korea. Both the large-scale and small-scale channel statistics were characterized: the large-scale parameters include the distance and frequency dependency of path loss and the shadowing fading statistics; the small-scale parameters include the distribution of clusters and MPC arrivals. 
The models found in the literature often refer to residential and office environments and the most general ones, that may be tuned to different environments, need specific parameters of the same environment, and thus complex measurements. Furthermore, statistical models involving channel response require time domain measurements and processing with high performance equipment. The attention here is on a simple, affordable and robust method based on frequency domain measurements and on the evaluation of the deviation from the free space loss law. The free space loss law says that the power density decreases with the square of distance from the source and it is typical of the far field propagation assumption.

\section{PROBLEM STATEMENT}

The target environment is that of a cruise ship and the publicly accessible spaces, in particular halls and corridors. Halls are characterized by one or two decks clearance, large surfaces (more than $1000 \mathrm{~m}^{2}$ ), several scattering objects such as tables, chairs and columns. Corridors have a variable width between 1 and $1.5 \mathrm{~m}$, depending on the arrangement of cabin doors, passenger ways and service doors, and behave like a large waveguide; the ceiling is not flat and contains edges and ridges, where lighting is located.

The present analysis is justified since, not only these two environments are representative of the public spaces on board, where wireless services are mostly required, but also they are also characterized by extensive use of steel for walls, floor and ceiling and aluminum for doors, handrails, panels, etc. Moreover, this electromagnetic environment has shown to be peculiar and never investigated before to authors' knowledge.

The analyzed characteristics are in relationship with a straightforward deterministic model of channel path loss for wireless services in the $2.4 \mathrm{GHz}$ frequency band. The attention is on the average signal strength for different distances and positions with respect to the transmitter, on the uniformity of the signal strength around a given measuring point and on the influence of common objects. The definition of this analysis scenario has been done keeping in mind the purpose of wireless facilities for telephone calls, messaging and entertainment.

Two open questions have been addressed by the foregoing analysis: what is the behavior of the path loss law? are the multipath terms significant and relevant to correct signal reception? The expected path loss law for free space propagation is a power law with exponent two, directly related to the decay of the power density versus the distance from the source. Multipath terms may degrade signal quality while not reducing measured average power significantly, simply because the measured power is absolutely not uniform across a section orthogonal to the direction of arrival of the main ray from the signal source.

\section{MEASUREMENT SYSTEM}

The measurements have been performed in the frequency domain using different measuring setups, to evaluate the relevance of multipath terms. To this aim three different techniques have been used:
1) a omni-directional antenna driven by an Access Point (AP) locked onto an 802.11 channel (channel 6 at $f_{0}=2437$ $\mathrm{MHz})$ with transmitter power $P_{t}=100 \mathrm{~mW}(20 \mathrm{dBm})$ and Orthogonal Frequency Division Multiplexing (OFDM) modulation (cycling over channel bandwidth among clearly distinguishable narrowband components, for the use with a spectrum analyzer); the receiver is either

1a) a laptop equipped with a wireless card and a diagnostic software (named in the following Laptop Analyzer, LA) or

1b) a Spectrum Analyzer (SA) connected to an omnidirectional antenna reading the received power at $f_{0}$ with a max-hold setting and five minutes dwell time (the time required to cycle over an OFDM frequency pattern);

2) a directive antenna (log-periodic antenna) fed by a RF generator sourcing a $2.5 \mathrm{GHz} 6 \mathrm{dBm}$ sinusoidal tone; the received signal is read for vertical and horizontal polarizations using the same S.A. and an omni-directional antenna; the source has smaller directivity to better understand the channel behavior with respect to multipath components.

Two different omni-directional antennas have been used: i) a custom sleeve dipole, obtained from a low loss coaxial cable, by cutting a piece of the insulating cover and folding down a length $l$ of the shield, thus leaving uncovered an equal length of the inner conductor; the length is tuned to the frequency $f_{0}$ so that the gain and the input impedance may be computed a priori [14]; ii) a wireless antenna for use with Access Points, that is a matched whip antenna (a helix antenna in the broadside operating mode) with a radiation patter and a gain very similar to that of a dipole [15].

For the considered wavelength $\lambda \cong 8 \mathrm{~cm}$, the positioning of the antenna system and the clearance around the antenna itself are two critical aspects, with respect to: first, electromagnetic field uniformity over a vertical section orthogonal to the direction of arrival (the line joining the transmitter and the receiver); second, the multipath fading phenomenon, influenced also by the measuring equipment and operator themselves. For this reason the measurement procedure requires at each measuring location that the spectrum analyzer and the operator are at least $3 \mathrm{~m}$ on the opposite side of the transmitter (this applies to the methods $1 \mathrm{~b}$ and 2). The same thing cannot be ensured when the laptop and its internal $\mathrm{WiFi}$ antenna are used (method 1a) and in this case the presence of the laptop itself must be considered. The main characteristics of the measuring equipment are [14][15]:

- $\quad$ the log-periodic antenna has a gain of $6.79 \mathrm{dBi}$ at 2.5 $\mathrm{GHz}$, while the omni-directional antennas have a 2.2 $\mathrm{dBi}$ gain; the results obtained with the omni-directional dipole antenna have been corrected for the input impedance and related reflection coefficient;

- the spectrum analyzer is battery supplied and all the uncertainty terms related to input reflection coefficient, input attenuator, linearity and internal noise are well within the observed variability of the received electromagnetic field. 
The uncertainty related to the measuring equipment and to the determination of calibration factors is reported in the following.

Method 1a). The laptop internal wireless antenna is considered equivalent to a dipole for directivity (and gain). The remaining measurement chain has no certificate or datasheet, thus it is considered matched and calibrated within an acceptable uncertainty of few $\mathrm{dB}$, confirmed by the readings on another laptop placed in the same position (substitution method).

Methods 1b) and 2). The S.A. is an Agilent mod. N1996A, for which the manufacturer datasheet and the calibration certificate state:

- displayed average noise level $-152 \mathrm{dBm} / \mathrm{Hz}$; for the used Resolution Bandwidth $(200 \mathrm{kHz})$ the displayed level was always more than $20 \mathrm{~dB}$ below the measured input signal, with a negligible contribution to overall uncertainty;

- $\quad$ overall amplitude accuracy (95\% confidence) $\pm 0.6 \mathrm{~dB}$ (this is the main accuracy contribution);

- scale fidelity $\pm 0.2 \mathrm{~dB}$ for the measured input levels.

The two small antennas have similar radiation patterns by theory (and thus similar gains) and the different input impedance and thus reflection coefficient has been numerically corrected during post-processing. The uncertainty of this correction has been extrapolated by accounting for frequency (with respect to $f_{0}$ ) and antenna geometry deviations, and the result is smaller than $1 \mathrm{~dB}$.
The directive antenna is a Scwarzbeck mod. 9134 logperiodic antenna, for which the antenna factor and gain are not accompanied by an explicit accuracy statement on the manufacturer datasheet. The calibration methods are different and it is known that they may differ so that a worst case uncertainty of $\pm 4 \mathrm{~dB}$ may be foreseen [16].

The used cable is a low loss RG400 and the length of the single piece is less than $2 \mathrm{~m}$, so the cable attenuation and related accuracy may be neglected.

Finally, as it will be shown by inspection of the results in the next Section, the data dispersion due to changes of antenna position and the effects of multipath fading is much larger than the equipment uncertainty.

\section{MEASUREMENT Results}

The presented results are aimed at first, validate the measurements obtained with either of the three methods by direct comparison, and, second, derive a law of propagation for the environments characteristics of a cruise ship, corridors and large halls.

\section{A. Large space: restaurant hall}

Measurements were taken at a restaurant hall located on two decks, with the AP located on the upper deck, close to the main entrance (see Fig. 1, where the AP label is repeated for reference also on the lower deck on the right).
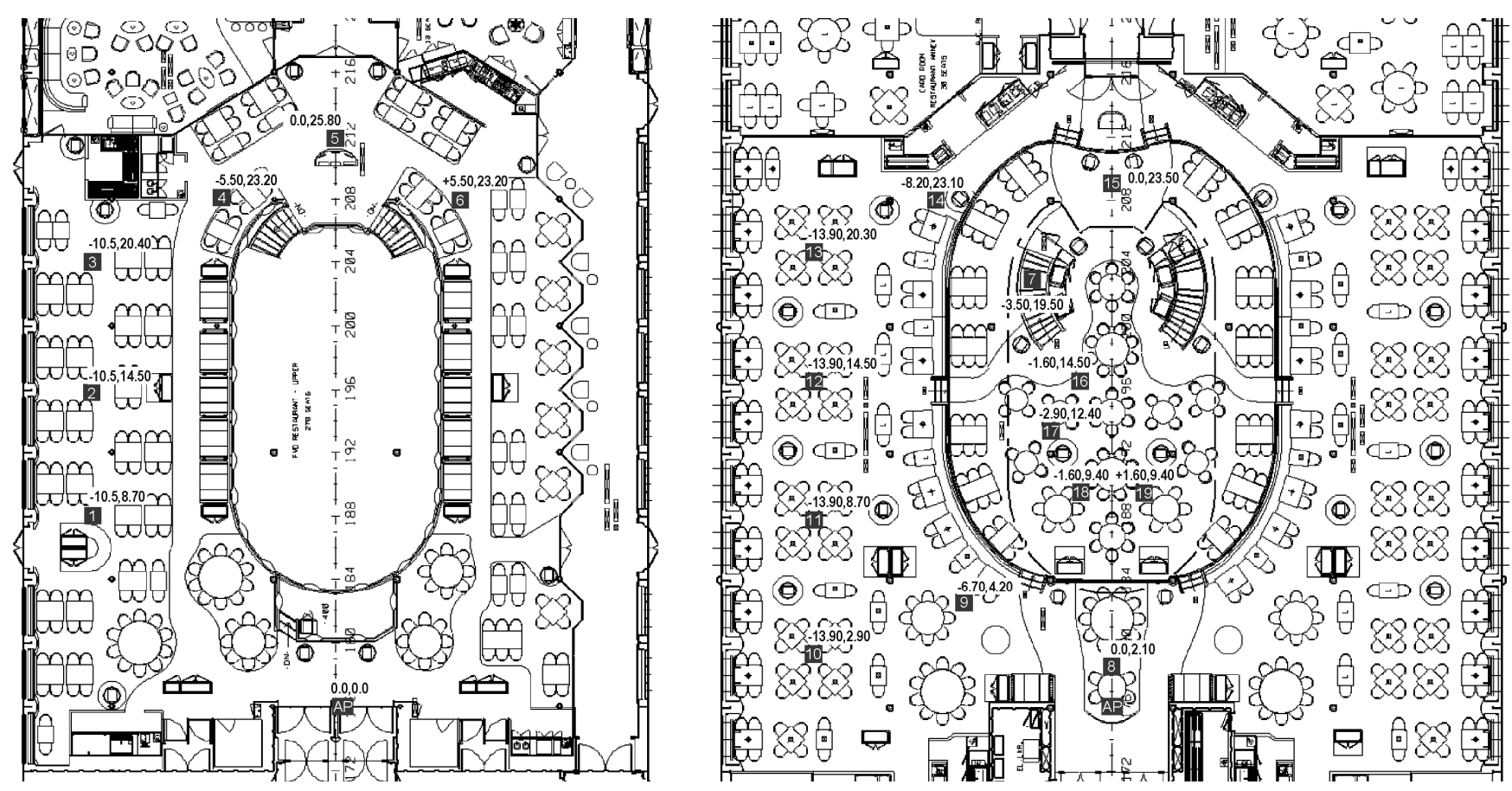

Figure 1. Map of the measurement points (black squares) in the restaurant hall (upper deck, left, and lower deck, right); the horizontal and vertical distance of each point from the AP are shown in metres. 
The power density values, recalculated by applying gain, effective aperture and corrective factors to the measured power at the points 1 through 19, are shown in Fig. 2.

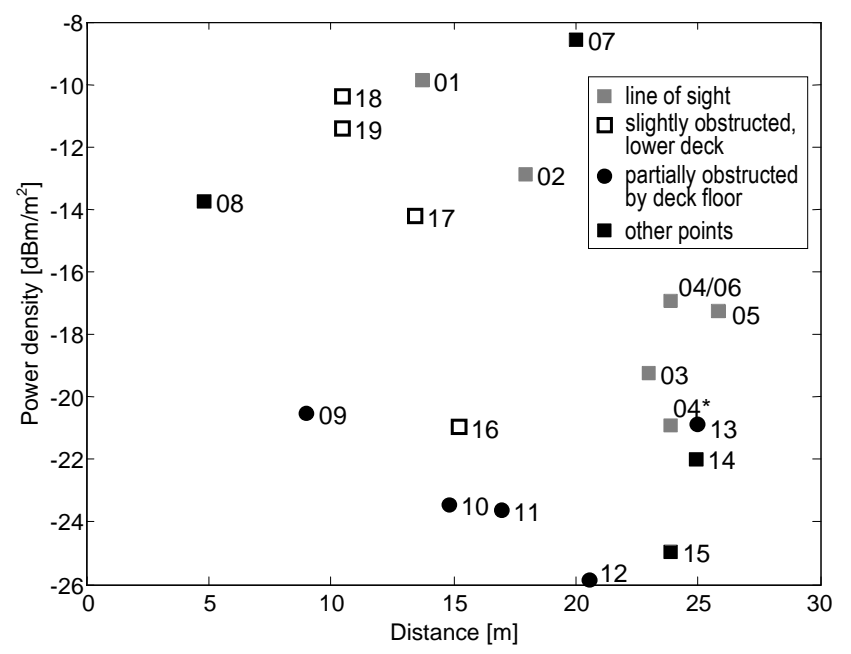

Figure 2. Power density values vs. distance (method $1 \mathrm{~b}$ );

the numbers refer to the measuring points of Fig. 1.

Comments on the results and measurement points of Fig. 2:

- the points 01, 02, 03 and 04 show a decay with distance that is almost corresponding to the expected free space path loss decay; going from point 1 to point 2 , the distance is increased by approximately $20 \%$ and the power density reduction is exactly $3 \mathrm{~dB}$ (the reciprocal of the square of the $20 \%$ increase is $1 / 1.44=0.69$ that is a $31 \%$ reduction, so $3 \mathrm{~dB}$ ); going from point 01 to point 03 the distance is increased by $60 \%$ and the power density reduction is almost $9 \mathrm{~dB}$, larger than the expected $4 \mathrm{~dB}$ because of increased scattering due to interposed columns and handrails; for points 04,05 and 06 the attenuation is only $1 \mathrm{~dB}$ larger than the expected free space loss (approximately $5 \mathrm{~dB}$ );

- point 08 , immediately below the AP, at a short distance but separated by a deck floor (light type), exhibits a power density larger than the one for a continuous heavy steel deck;

- points 04 and $04 *$ : while measuring point 06 , it was clear that the scattering of a column in front of former point 04 (then called 04*) had a relevant effect; the measurement was repeated for a really symmetrical position obtaining a new almost identical value;

- points 10,11, 12 and 13: even if these points are in a similar sequence and spatial location as points 01,02 , 03 and 04 , they don't lie on the upper deck of the AP and because of the presence of the deck floor the received power density is lower; at point 13 , the lineof-sight is again ensured and the received power density increases to a value closer to the one measured for similar points 04 and 06 ;

- points 16, 17, 18 and 19 are points in front of the AP with different situations of obstacles and angles: while 17, 18 and 19 almost meet the free space losses law, point 16 is experiencing partial obstruction of a column, with a loss of $7 \mathrm{~dB}$ with respect to point 17;

- point 07 midway between the two decks is the best position for line-of-sight view of the AP without interfering objects.

\section{B. Narrow space: cabins corridor}

The analysis of the measurements taken in a cabins corridor revealed a significant multipath reflection and a peculiar path loss law. Measurements were taken at two different heights above ground $(1 \mathrm{~m}$ and $1.5 \mathrm{~m})$ and three horizontal positions (centre of the corridor and $\pm 0.4 \mathrm{~m}$ from the centre, with a minimum distance from the walls of $0.2 \mathrm{~m}$ ), as shown in Fig. 3.

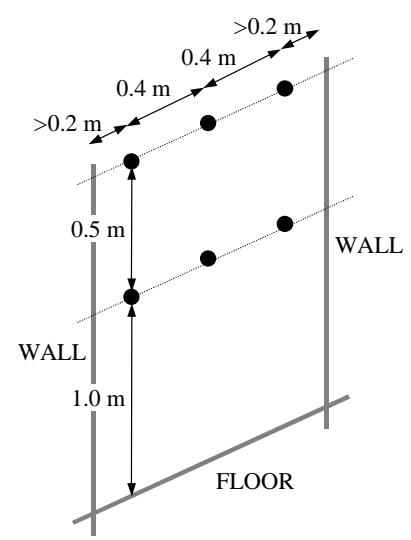

Figure 3. Measurement points at each measurement location

In Fig. 4 all the measurement results are collected together as robust averages (by discarding $33 \%$ of the outlying values) with dispersion intervals, determined as the sample deviation standard on the set of all measurements at each location.

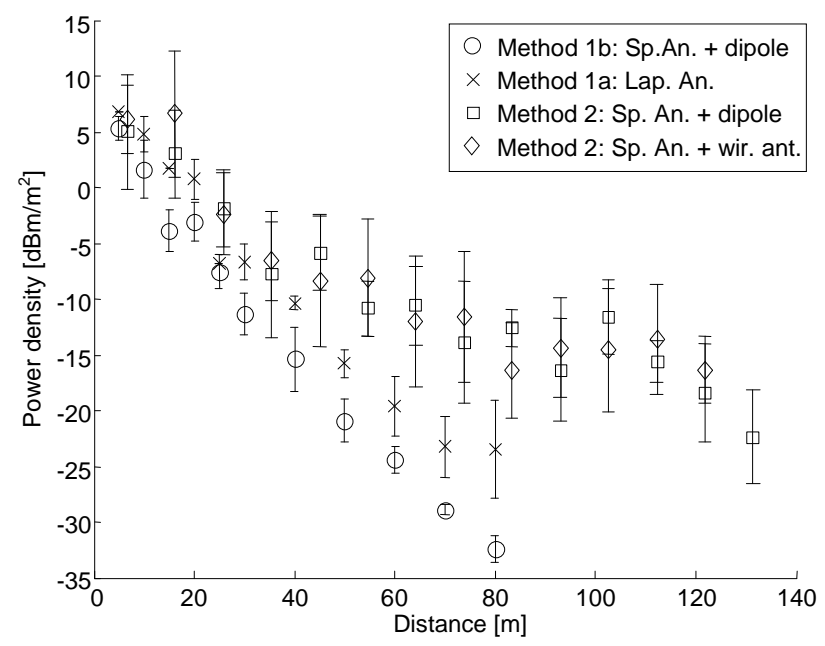

Figure 4. Average power density vs. distance (vertically polarized field)

From Fig. 4 we see that the data dispersion is larger for omni-directional sourcing (obtained with the AP equipped with its own antenna). At some distances data dispersion is comparable also for the more directive method 2, but this is due to the specific geometry of the measurement location (hand rails, edges, more doors). 
The curves shown in Fig. 4 are separated for the used measurement methods. The attenuation curve (or path loss) is quite different for the two methods (see Fig. 5 below), since in the first the multipath influence is much more significant, while the log-periodic antenna is able to convey the emitted power into a smaller solid angle, thus avoiding a large part of multipath interference in the first part of the corridor. Of course, the geometry of the corridor is peculiar and characterized by a $25 \mathrm{~cm}$ enlargement of the corridor width (normally $1 \mathrm{~m}$ ) at the cabin doors and the presence of handrails and several service doors, some of which locked open at the moment of the measurements; moreover the ceiling is not homogeneous and is full of edges and niches for lighting. For this reason some of the 6 measuring points at each longitudinal position may give a much lower (or higher in some cases) reading than the others, since exposed to the influence of operator's body and its position besides location geometry; from this the need for robust statistical analysis and removal of outliers during data post-processing.

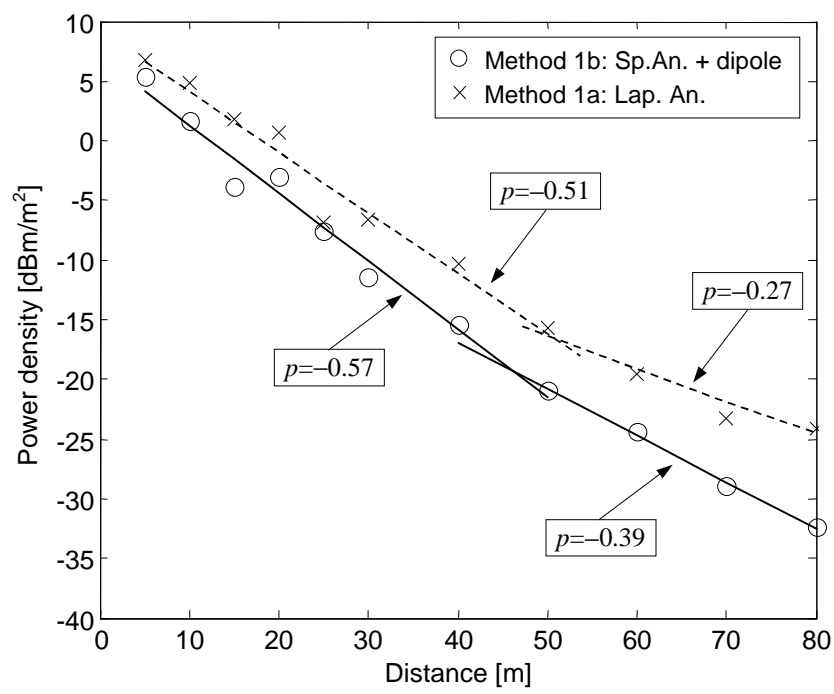

(a)

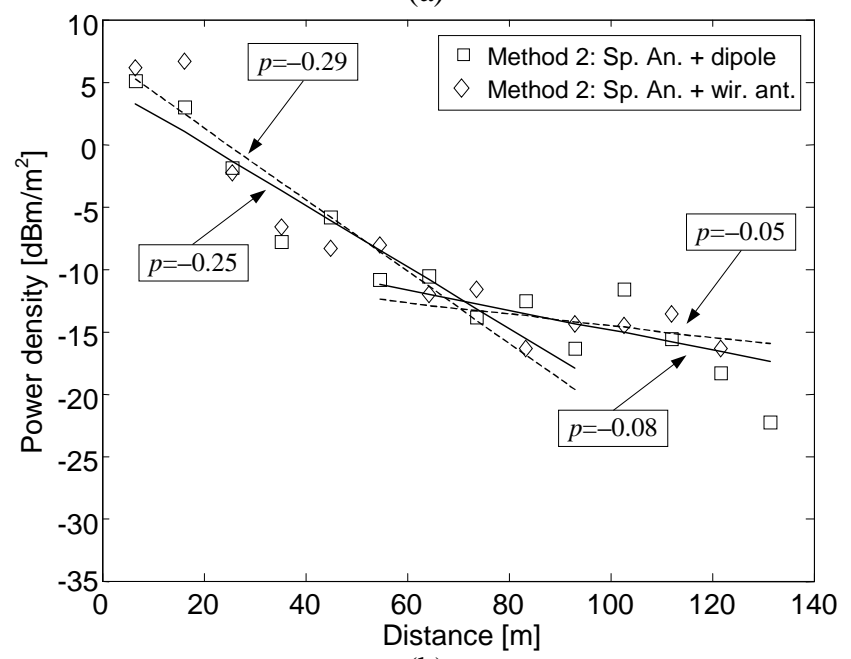

(b)

Figure 5. Average power density values vs. distance and linear interpolation by least mean square regression: (a) omni-directional sourcing (method 1), (b) directional sourcing (method 2$)$
From Fig. 5(a) we see that the S.A. and the L.A. values are in agreement up to about 60-70 $\mathrm{m}$ distance, with the L.A. values larger than S.A. values by what we could call "multipath gain": the S.A. method 1b) measures exclusively the central frequency component using max hold and repeated sweeping over the $f_{0}$ frequency interval, thus excluding multipath components, while the received power calculated by method 1a) is based on the acquisition of the complete channel bandwidth and demodulation of all the signal components. The difference between L.A. and S.A. values ranges from few $\mathrm{dB}$ up to $6 \mathrm{~dB}$, with the largest difference above about 30-40 m.

Above 60-70 m S.A. values (method $1 \mathrm{~b}$ ) decreases more rapidly than L.A. values (method 1a); a possible explanation may be found in the transversal corridor at $65 \mathrm{~m}$ distance that probably largely modifies the propagation along the main corridor, so that the multipath terms become a significant percentage of the received signal. The difference between L.A. and S.A. values increases slightly from 6 to $8 \mathrm{~dB}$.

It is possible to exemplify the power density decay vs. distance as:
$10 \mathrm{~m} \rightarrow 25 \mathrm{~m}:$
$10 \mathrm{~dB}$ of attenuation
$25 \mathrm{~m} \rightarrow 50 \mathrm{~m}:$
$10 \mathrm{~dB}$ of attenuation
$50 \mathrm{~m} \rightarrow 75 \mathrm{~m}$
$10 \mathrm{~dB}$ of attenuation

This behavior is rather peculiar, since it doesn't meet any power-law profile, as it would be expected.

From Fig. 5(b) we see that the slope of the path loss is approximately one half of the previous results and that above $70 \mathrm{~m}$ the interpolating line is almost horizontal, to confirm that the direct and reflected rays have found a favorable propagation mechanism, possibly thanks to the more favorable angle of incidence of the rays emitted by the directive source.

The directive generation of wireless signals represents thus a valid method for the wireless coverage of long reflecting and diffracting corridors.

Concerning the extension of the wireless coverage inside cabins, it was experimentally determined a power loss of about $10 \mathrm{~dB}$ from the outside to the inside due to the cabin door, that may be easily taken into account during coverage study with a suitable margin on the calculations.

\section{CONCLUSIONS}

A preliminary analysis of the propagation of wireless signals on board cruise ships is presented. The environment is rather peculiar since there are numerous reflecting surfaces and several areas are characterized by a large scattering due to metallic columns, handrails, niches, doors, edges and corners both on walls and ceilings. The characterization of the wireless channel has been done in the frequency domain for large and narrow spaces, namely a restaurant hall and a cabin corridor. Large areas may be characterized by an almost free space law of propagation, while a non-power law of propagation has been found for the corridor.

The measurement setup is simple and easy to position and operate; different antennas may be used to select the amount of multipath components for a given environment geometry (corridor length or hall extension). The results consist of 
corrected path loss laws for halls and corridors and the quantification of scattering influence. Multipath components as well as the path loss versus distance may be kept under control in the final application if a directive antenna is used as the source antenna of the Access Point.

Another outcome of the measurement campaigns and processing of the measurement results is a simplified tool for the planning of wireless network coverage, that is the assignment of the number and position of Access Points with respect to the geometry of the environment. The tool incorporates the power decay curves, the characteristics of the sourcing and receiving hardware and the margins due to measurement uncertainties and second order effects.

\section{REFERENCES}

[1] T. S. Rappaport, Wireless Communications: Principles and Practice, $2^{\text {nd }}$ ed., Prentice Hall, Upper Saddle River, NJ, 2002.

[2] T. S. Rappaport and S. Sandhu, "Radio-wave propagation for emerging wireless personal communication systems," IEEE Antennas and Propagation Magazine, vol. 36, n. 5, pp. 14-24, Oct. 1994.

[3] B. Uguen, E. Plouhinec, Y. Lostanlen, and G. Chassay, "A deterministic ultra wideband channel model," in Proc. IEEE Conf. UWB Systems and Technologies (UWBST02), Baltimore, MD, May 2002, pp. 1-6.

[4] R. Yao, W. Zhu, and Z. Chen, "An efficient time-domain ray model for UWB indoor multipath propagation channel," in Proc. IEEE Vehicular Technologies Conf. (VTC 2003-Fall), Orlando, FL, September 2003, pp. 1293-1297.

[5] R. Yao, G. Gao, Z. Chen, and W. Zhu, "UWB multipath channel model based on time domain UTD technique," in Proc. IEEE Global Telecommunications Conf. (GLOBECOM03), San Francisco, CA, December 2003, pp. 1205-1210.
[6] H. Sugahara, Y. Watanabe, T. Ono, K. Okanoue, and S. Yarnazaki, "Development and experimental evaluations of "RS-2000"propagation simulator forUWBsystems," IEEE Conf. UWB Systems and Technologies (UWBST04), Kyoto, May 2004, pp. 76-80.

[7] A. Armogida, B. Allen, M. Ghavami, M. Porretta, G. Manara, and H. Aghvami, "Path loss modelling in short range UWB transmissions," Int. Workshop on Ultra Wideband Systems (IWUWBS03), Oulu, June 2003.

[8] S. S. Ghassemzadeh, R. Jana, C. Rice, W. Turin, and V. Tarokh, "Measurement and modeling of an ultra-wide bandwidth indoor channel," IEEE Trans. on Communications, vol. 52, n. 10, pp. 17861796, Oct. 2004.

[9] J. R. Foerster and Q. Li, "UWB channel modeling contribution from Intel,” IEEE P802.15-02/279r0-SG3a, June 2002.

[10] C.-C. Chong, Y. Kim, and S.-S. Lee, "Statistical characterization of the UWB propagation channel in various types of high-rise apartments," in Proc. IEEE Wireless Communications Networking Conf. (WCNC05), New Orleans, LA, March 2005, pp. 944-949.

[11] C.-C. Chong, Y. Kim, S. K. Yong, and S.-S. Lee, "Statistical characterization of the UWB propagation channel in indoor residential environment," Wireless Communications and Mobile Computing, (Special Issue on UWB Communications), Aug. 2005, vol. 5, no. 5, pp. 503-512.

[12] C.-C. Chong and S. K. Yong, "A generic statistical based UWB channel model for high-rise apartments," IEEE Trans. Ant. Propagat., vol. 53, no. 8, pp. 2389-2399, Aug. 2005.

[13] C.-C. Chong, Y. Kim, and S.-S. Lee, "UWB channel model for indoor residential environment," IEEE 802.15-04-0452-01-004a, Sept. 2004.

[14] C. Balanis, Antenna Theory: Analysis and design, 2nd ed., John Wiley

[15] R.C. Johnson (ed.), Antenna Engineering Handbook, McGraw-Hill, 3rd ed., 1993.

[16] A. Mariscotti, "Measurement Procedures and Uncertainty Evaluation for Electromagnetic Radiated Emissions from Large Power Electrical Machinery", IEEE Trans. on Instrumentation and Measurement, vol. 56 n. 6 , Dec. 2007, pp. 2452-2463. 